\title{
Pseudo Mean Baseline Removal Method
}

National Cancer Institute

\section{Source}

National Cancer Institute. Pseudo Mean Baseline Removal Method. NCI Thesaurus. Code C64242.

Method for identifying invariant set normalization baseline using psuedo mean

expression values. Defined in the documents available at

http://bioconductor.org/packages/1.9/bioc/html/affy.html. 